\title{
Reply to Comments on SPSSI Research Summary on Media Violence by Cupit (2016), Gentile (2016), Glackin \& Gray (2016), Gollwitzer (2016), and Krahé (2016)
}

Brad J. Bushman

The Ohio State University \& VU University Amsterdam

Craig A. Anderson

lowa State University

Edward Donnerstein

University of Arizona

Tom. A. Hummer

Indiana University School of Medicine

Wayne Warburton

Macquarie University

\begin{abstract}
Author Notes
Correspondence concerning this article should be addressed to Brad J.

Bushman, School of Communication, The Ohio State University, 3016 Derby Hall, 154 North Oval Mall, Columbus, OH, USA. Email: bushman.20@osu.edu. Analyses of Social Issues and Public Policy, in press (June, 2016).
\end{abstract}

This is the author's manuscript of the article published in final edited form as:

Bushman, B. J., Anderson, C. A., Donnerstein, E., Hummer, T. A., \& Warburton, W. A. (2016). Reply to Comments on SPSSI Research Summary on Media Violence by Cupit (2016), Gentile (2016), Glackin and Gray (2016), Gollwitzer (2016), and Krahé (2016). Analyses of Social Issues and Public Policy, 16(1), 443-450. http://dx.doi.org/10.1111/asap.12123 


\begin{abstract}
In responding to the published comments on our SPSSI Research Summary on Media Violence, we note that several key themes emerge. In assessing the media violence research evidence it is more informative and less biased to draw conclusions based on the full range of findings than to emphasize findings from individual studies. Using the full range of studies, it is clear that consuming violent media influences the way people think and feel, and increases the likelihood of aggressive behavior. However, when placing such findings into real world settings, it is important to consider media violence exposure as one of many risk factors for violence and aggression rather than as a sole factor. This acknowledgement of multiple causal factors does not make media violence unimportant - it is one of the few risk factors for aggression that can be addressed relatively easily and inexpensively. To this end, researchers are encouraged to now focus their efforts on finding those factors that moderate the media violence exposure-aggression link, and policy makers and professionals who work with children are encouraged to incorporate media violence science into their practices and decision-making.
\end{abstract}




\section{Reply to Comments on SPSSI Research Summary on Media Violence by Cupit (2016), Gentile (2016), Glackin \& Gray (2016), Gollwitzer (2016), and Krahé (2016)}

We would like to thank Cupit (2016), Gentile (2016), Glackin and Gray (2016), Gollwitzer (2016), and Krahé (2016) for their thoughtful comments on our Research Summary on Media Violence (Anderson, Bushman, Donnerstein, Hummer, \& Warburton, 2015). We are delighted that this important summary has attracted attention from these scholars, and we respond to their major points in this article.

\section{Evidence Aggregated Across Many Studies is More Informative than \\ Evidence From Individual Studies}

When it comes to scientific evidence, we believe that it is important to look at the whole forest rather than isolated trees. In this analogy, the forest is the collection of all studies, and the trees are the individual studies. One common strategy researchers use is to choose their favorite tree and then claim it represents the entire forest. Often their favorite tree is the study that they, their student, or their colleague conducted. Because there are lots of trees to choose from, researchers can criticize conclusions drawn from a 'forest' of research by finding the most poorly conducted study they can find from the tail of the distribution and selectively point out its flaws. They can also choose the most rigorous study that confirms their own hypothesis and point out its many strengths. 
All people, including researchers, can be biased in their search for evidence. However, such bias can be minimized when analyses of findings use all the evidence and thus provide a view of the entire forest. Because wellconducted meta-analytic reviews include all of the available findings, our research summary draws its conclusions from several large meta-analytic reviews of violent media effects rather than from a selection of our favorite studies.

As Gollwitzer (2016) notes, cumulative evidence is what leads to scientific progress. Meta-analytic reviews provide cumulative evidence. Cumulative evidence becomes even more persuasive when it is consistent across different types of studies, different types of measures of concepts, different types of participant samples, different countries, and different laboratories. Scientists call this convergence of evidence "triangulation." As our research summary shows, this triangulation of evidence definitely occurs for media violence studies.

\section{Media Violence is Not the Only or Even the Most Important Risk Factor for Violence and Aggression}

In the wake of a mass shooting, people want to know "the" cause of the shooting, and exposure to violent media is often singled out as "the" cause. However there is never a single cause. Mass shootings are rare and complex events, and are caused by multiple factors acting together. Thus it is essentially impossible to predict a mass shooting based on exposure to violent media or any other single factor. When it comes to rare acts of violence (e.g., mass shootings, murders, assaults), exposure to violent media may play a small role, along with 
the other risk factors, but it is never both necessary and sufficient to cause violent behavior alone. Of course, the same is true of other risk factors.

Importantly, when it comes to more common aggressive behaviors (e.g., bullying, teasing, ostracizing, rumor-spreading), fewer risk factors are necessary to instigate them, and exposure to violent media can play a much larger role, a point made by Gentile (2016). As noted in our original summary, and as Gollwitzer (2016) and Gentile (2016) note in their comments, exposure to media violence is not the only risk factor for aggression and violence - it is one of many risk factors.

Some early models proposed that exposure to violent media had a direct, immediate, and powerful effect on viewers, like a drug injected into one's bloodstream. Modern theories (e.g., General Aggression Model: Anderson \& Bushman, 2002; Social cognitive theory: Bandura, 1986; Script theory: Huesmann, 1986) are far more complex and propose several factors that can moderate and mediate violent media effects. As Gentile (2016) notes, "even the strongest critics of the literature find that media violence has effects on thoughts and feelings (e.g., Ferguson, 2007a). This alone should make the complexity apparent. We all know that our thoughts and feelings are related to our behaviors, but not in a simple mechanistic way. This is why the effect of media violence, or almost any experience for that matter, does not relate in a simple mechanistic way to any given outcome" (p. 3).

As Glackin and Gray (2016) note, risk and protective factors often combine in complex ways throughout the course of a child's development. 
“(M)ultiple risk factors do not simply add up like weights hung around a child's neck" (Coie, Miller-Johnson, \& Bagwell, 2000, p. 100).

Yet it cannot be denied that exposure to media violence is a risk factor for aggression and violence. How could it not be, theoretically or practically? For decades both therapists and researchers have argued that observing violence increases the likelihood of a child being aggressive, whether they observe it in the home, at school, or in their community. Why, then, would observing violence in the mass media not have the same impact? Of course there is a difference between the virtual world and the real world, but we can think of no theory that would predict that exposure to violent media should not impact the way children think, feel, and behave.

In practical terms, most people today are immersed in the media, like fish in water. American children $8-18$, on average, spend more than 7.5 hours per day consuming mass media (Rideout, Foehr \& Roberts, 2010), more time than they spend in school. Recent studies show that American adults may spend as much or more time consuming media (e.g., Short, 2013). Violence is a dominant theme in many forms of media (e.g., television, film, Internet, video games, music). We can think of no activity that people can engage in for over 7 hours per day that would have no effect on the way they think, feel, and behave. After all, the human brain is plastic, and its structure is shaped on multiple levels by what we experience (May, 2011). Indeed, as Gentile (2016) notes, people expect to be affected by the media: "If we choose to watch a violent film or play a violent 
video game and it truly has no effect on us, we call it 'boring' and stop watching it" (p. 3).

It is important to point out that exposure to media violence is also one of the few risk factors for aggression and violence that policy makers, professionals, and parents can actually do something about, and for little cost. Other risk factors for aggression and violence are much more costly and difficult (or even impossible) to change, such as being a male, having low intellectual functioning, living in poverty, prenatal (maternal) malnutrition, being exposed to family violence, and alcohol and drug addiction.

\section{Moderators of Violent Media Effects}

In our view, we need to move beyond the question of whether exposure to violent media is a risk factor for aggression and violence (also see Cupit, 2016), and focus instead on personal and situational variables that moderate violent media effects. One potential moderator is culture. We agree with Krahe that "violent media do not exist in a cultural vacuum" (p. 3). Although a recent metaanalysis found no difference in the magnitude of effects obtained from Eastern and Western countries (Anderson et al., 2010), most media violence studies are conducted in the United States. The majority of violent media (e.g., films, video games) also come from the United States. One writer pointed out that violent media might be one of the most exportable commodities from the U.S., making the U.S. "bread-casket for the world" (Hammerman, 1990, p. 79). Media violence "sells" extremely well across cultures (e.g., Hamilton, 1998). However, saturation levels of violent media differ across cultures. Also, violence is interpreted 
differently through different cultural lenses. Thus, more media violence studies are needed from outside the U.S. For example, it would be interesting to test whether participants from individualistic cultures are more affected by violent video games played alone, and if participants from collectivist cultures are more affected by violent video games played with others.

In addition to cultural contexts, violent media effects should be examined within family contexts. Although we did not discuss the developmental psychopathology approach in our summary, we agree with Glackin and Gray (2016) that it is a promising approach for understanding how violent media may affect children, especially those at risk for aggression and violence.

Other possible moderators include features of the violent content itself (Gentile, 2016), such as the presence of blood, degree of realism, moral context, portrayal of consequences of violence, whether the game is played alone or with others, and where attention is focused (among others). We agree that violence is not a unidimensional construct (e.g., Adachi \& Willoughby, 2011), and that players might have different motivations for consuming violent media (e.g., Ryan, Rigby, \& Przybylski, 2006).

\section{Interventions}

Violent entertainment has always been part of human history, from gladiator games to public executions. But today violent entertainment is more accessible than ever before. Violent entertainment is not going to vanish. Thus, the question is what to do about it. We offered some suggestions in our initial summary, and those who commented on our summary have offered some 
additional suggestions.

We discussed the concept of media literacy in our initial summary, and Gollwitzer (2016) also mentioned it in his comment. Media literacy programs can help children become more intelligent and critical media consumers (e.g., Bickham \& Slaby, 2012), and can even help reduce aggression and violence levels. In one study, for example, middle school students who were randomly assigned to participate in a violent media literacy program were 2.16 less likely to push or shove another student and were 2.32 times less likely to threaten to hit or hurt someone in comparison to control students (Fingar \& Jolls, 2014). However, media literacy programs differ greatly in many ways, and most do not have an appreciable effect on later aggressive behavior. More research on "what works" is badly needed.

We also agree with Gollwitzer (2016) that more research is needed on other protective factors that might make children more resilient to violent media effects. As Glackin and Gray (2016) note, caregivers play the most important role in protecting and buffering children from the harmful effects of violent media.

As Cupit (2016) notes, it seems like "overkill" to produce yet another statement about violent media effects, but we hope that through repetition policy makers, professionals, and parents will eventually "get the message" that exposure to violent media is a risk factor for aggression and violence. It is not the only risk factor, or even the most important risk factor, but it is a non-trivial risk factor and certainly one that can be addressed. 


\section{References}

Bickham, D. S., \& Slaby, R. G. (2012). Effects of a media literacy program in the us on children's critical evaluation of unhealthy media messages about violence, smoking, and food. Journal Of Children And Media, 6(2), 255271. doi:10.1080/17482798.2012.662031

Ferguson, C. J. (2007a). Evidence for publication bias in video game violence effects literature: A meta-analytic review. Aggression and Violent Behavior, 12(4), 470-482. doi:10.1016/j.avb.2007.01.001

Fingar, K. R., \& Jolls, T. (2014). Evaluation of a school-based violence prevention media literacy curriculum. Injury Prevention, 20(3), 183-190. doi:10.1136/injuryprev-2013-040815

Hamilton, J. T. (1998). Channeling violence: The economic market for violent television programming. Princeton, NJ: Princeton University Press.

Hammerman, J. K. (1990, Oct.). Dead men don't smirk. Esquire, v114, n4, p79(1).

May, A. (2011). Experience-dependent structural plasticity in the adult human brain. Trends in Cognitive Sciences, 15(10), 475-482. doi:10.1016/j.tics.2011.08.002

Rideout, V. J., Foehr, U. G., \& Roberts, D. F. (2010). Generation M2: Media in the lives of 8-18 year olds. Merlo Park CA: Henry J Kaiser Foundation.

Short, J. E. (2013). How much media? 2015 report on American consumers. University of Southern California. Retrieved from http://www.marshall.usc.edu/faculty/centers/ctm/research/how-muchmedia 